INPLASY

PROTOCOL

To cite: Zhong et al. Effect of Hydrogen gas on Acute Lung Injury in sepsis mice: a systematic review and metaanalysis. Inplasy protocol 202120033. doi:

10.37766/inplasy2021.2.0033

Received: 09 February 2021

Published: 09 February 2021

Corresponding author:

Guoshen Zhong

walshonzhong@gmail.com

Author Affiliation:

Weifang Medical University

Support: None.

Review Stage at time of this submission: Formal screening of search results against eligibility criteria.

Conflicts of interest:

None declared.

\section{Effect of Hydrogen gas on Acute Lung Injury in sepsis mice: a systematic review and meta-analysis}

Review question / Objective: P: Acute Lung Injury in sepsis mice. I: Using $2 \%$ hydrogen gas to treat the sepsis mice as the group of intervention 1h per day. C: Acute Lung Injury in sepsis mice as the group of comparison. $O$ : The number of dead mice in the experimental group and the control group. S:One reviewer (GSZ) generated the search strategy, searched the databases, and made a list of all the records. Two evalutors (XYD,YYZ) independently evaluated the articles based on the inclusion and exclusion criteria.Disagreements were solved together through discussions (GSZ,XXD,YYZ). Two reviewers (XYD,YYZ) independently extracted the data. Data were extracted: the last name of the author, publication year, animal model, species of mice, number of animal, the time of treatment and outcome. If provided several outcomes were presented, only extracted final outcomes. While the outcome data showed by graphically, tried to contact the authors to get the detailed data, otherwise, we used GetData software to measure the data.

INPLASY registration number: This protocol was registered with the International Platform of Registered Systematic Review and Meta-Analysis Protocols (INPLASY) on 09 February 2021 and was last updated on 09 February 2021 (registration number INPLASY202120033).

\section{INTRODUCTION}

Review question / Objective: P: Acute Lung Injury in sepsis mice. I: Using $2 \%$ hydrogen gas to treat the sepsis mice as the group of intervention 1h per day. C: Acute Lung Injury in sepsis mice as the group of comparison. O: The number of dead mice in the experimental group and the control group. S:One reviewer (GSZ) generated the search strategy, searched the databases, and made a list of all the records. Two evalutors (XYD,YYZ) independently evaluated the articles based on the inclusion and exclusion criteria. Disagreements were solved together through discussions (GSZ, XXD, YYZ). Two 
reviewers (XYD,YYZ) independently extracted the data. Data were extracted: the last name of the author, publication year, animal model, species of mice, number of animal, the time of treatment and outcome. If provided several outcomes were presented, only extracted final outcomes. While the outcome data showed by graphically, tried to contact the authors to get the detailed data, otherwise, we used GetData software to measure the data.

Condition being studied: (1)Subjects: Only included Acute Lung injury in Sepsis mice. (2)Intervention: Only Hydrogen gas therapy was included. (3)Outcome: The number of deaths in experimental and control mice (4)Language: Articles were published by English and Chinese were included. (5)No publication date limit was set, and the search was conducted in December 2020.

\section{METHODS}

Participant or population: Acute Lung Injury in sepsis mice.

Intervention: Using $2 \%$ hydrogen gas to treat the sepsis mice as the group of intervention $1 \mathrm{~h}$ per day.

Comparator: Acute Lung Injury in sepsis mice as the group of comparison.

Study designs to be included: One reviewer (GSZ) generated the search strategy, searched the databases, and made a list of all the records. Two evaluators (XYD,YYZ) independently evaluated the articles based on the inclusion and exclusion criteria. Disagreements were solved together through discussions (GSZ, XXD, YYZ). Two reviewers (XYD,YYZ) independently extracted the data. Data were extracted: the last name of the author, publication year, animal model, species of mice, number of animal.

Eligibility criteria: (1)Subjects: Only included Acute Lung injury in Sepsis mice. (2)Intervention: Only Hydrogen gas therapy was included. (3)Outcome: The number of deaths in experimental and control mice
(4)Language: Articles were published by English and Chinese were included. (5)No publication date limit was set, and the search was conducted in December 2020.

Information sources: The following databases were searched from database inception to December 2020: PubMed, Embase, Cochrane, Web of science, Ovid Medline and CNKI.

Main outcome(s): Outcome: The number of deaths in experimental and control mice.

Additional outcome(s): None.

Data management: Data were extracted:the last name of the author, publication year, animal model, species of mice, number of animal, the time of treatment and outcome.if provided several outcomes were presented, only extracted final outcomes.While the outcome data showed by graphically, tried to contact the authors to get the detailed data, otherwise, we used GetData software to measure the data.

Quality assessment / Risk of bias analysis: We evaluated methodological quality against SYRCLE (Systematic Review Centre for Laboratory animal Experimentation). and each parameter for the type of bias was scored as low, high, or unclear risk of bias. Two evaluators (XXD, YYZ) independently extracted the data and assessed the quality of each study. Disagreements Were solved by one evaluator (GSZ).

Strategy of data synthesis: The following key words were used: Sepsis, Spetic shock,Acute lung injury, Hydrogen and molecular hydrogen. The search languages were limited to English and Chinese, and collect records from other sources.

Subgroup analysis: According to the types of mice, they were divided into C57BL / 6 and ICR groups.

Sensitivity analysis: Not, because all of the studies are less than 10 , according to the 
hand book of Cochrane, we didn't do sensitivity

Language: English and Chinese.

Country(ies) involved: China.

Other relevant information: None.

Keywords: Hydrogen gas, ALI, Sepsis, Meta-analysis, Systematic evaluation.

Dissemination plans: None.

Contributions of each author:

Author 1 - Guoshen Zhong - The author generated the search strategy, searched the databases, and made a list of all the records.

Author 2 - Xinye Deng - The author evaluated the articles based on the inclusion and exclusion criteria, in addition, extracted the data from articles.

Author 3 - Yuanyun Zhang - The author evaluated the articles based on the inclusion and exclusion criteria, in addition, extracted the data from articles. 